\title{
Detection of a stellar halo in NGC 4244
}

\author{
A. Seth ${ }^{1}$, R. de Jong ${ }^{2}$, J. Dalcanton ${ }^{3}$, and the GHOSTS team \\ ${ }^{1}$ Harvard-Smithsonian Center for Astrophysics, 60 Garden St., Cambridge, MA 02139 \\ email: aseth@cfa.harvard.edu \\ ${ }^{2}$ Space Telescope Science Institute \\ ${ }^{3}$ University of Washington
}

\begin{abstract}
We present the preliminary detection of a stellar halo in the nearby, edge-on galaxy, NGC 4244. Using data from the Hubble Space Telescope's Advanced Camera for Surveys (HST/ACS), we have examined number counts of red giant branch (RGB) stars along the minor axis of the galaxy out to $10 \mathrm{kpc}$, with a limiting surface brightness of $\mu_{R} \sim 31 \mathrm{mag} / \mathrm{arcsec}^{2}$. At distances more than $2.5 \mathrm{kpc}$ above the disk, a second stellar component is clearly visible. This component is well fit by either an exponential or a power law. The best-fit exponential scale height is similar to the scale length of the galaxy disk, suggesting that we are indeed detecting a halo component. Furthermore, the color of the RGB stars above $2.5 \mathrm{kpc}$ are significantly bluer than those in the disk, suggesting a more metal-poor population. Outside the local group, this finding represents one of the first detections of a resolved stellar halo in a spiral galaxy.
\end{abstract}

The stellar halos of disk galaxies are thought to be made from merger remnants, and thus trace the earliest epochs of galaxy formation (e.g. Bullock \& Johnston 2005). However, due to their low surface brightnesses, stellar halos have been detected in only a small number galaxies, mostly in the Local Group using resolved stars (e.g. Kalirai et al. 2006; McConnachie et al. 2006). Complementing these studies, Zibetti et al. (2004) examine the integrated light profiles of $>1000$ stacked edge-on galaxies and show that stellar halos may be a common feature of disk galaxies.

Using HST, we can detect and study stellar halos around nearby $(\mathrm{D}<10 \mathrm{Mpc})$ galaxies using resolved RGB stars. NGC 4244 is a low mass $\left(V_{\text {rot }} \sim 100 \mathrm{~km} / \mathrm{sec}, M_{B}=-17.7\right)$, bulgeless spiral, and is one of the nearest edge-on galaxies $(\mathrm{D}=4.36 \mathrm{Mpc}$, Seth et al. 2005a). The galaxy has smaller dimensions than the Milky Way with a scale length of $1.8 \mathrm{kpc}$ and a scale height of $\sim 300 \mathrm{pc}$. We have obtained Cycle 14 HST/ACS snapshot images of seven fields along the principal axes of the disk (Fig. 1, left panel). In this contribution, we analyze the distribution and color of RGB stars along the minor axis.

Results: Figure 2 (left panel) shows the RGB surface density as a function of height above the midplane. The dip at low disk heights is caused by incompleteness. At larger disk heights, a clear exponential drop off is seen that matches the scale height found by Fry et al. (1999) using deep ground-based data with a limiting surface brightness $\mu_{R}=27.5$. We have used the Fry et al. (1999) data to calibrate our RGB surface density to $\mu_{R}$. Starting $\sim 2.5 \mathrm{kpc}$ above the plane at $\mu_{R}>28$, the RGB surface density starts declining more slowly. We have fit this 2nd component to both an exponential and a power-law, and find that both fit the data well. The best fitting exponential has a scale height of $\sim 1.6 \mathrm{kpc}$, nearly as large as the scale length of the NGC 4244 disk, while the best-fit power law falls off as $\sim z^{-2.3}$, similar to the Milky Way halo if seen in projection. Either fit suggests that the component we are detecting is a stellar halo. This component is much fainter than the Milky Way halo; integrating the best-fit exponential assuming a scale length similar to the disk suggests a mass of only $3 \times 10^{6} \mathrm{M}_{\odot}$. 

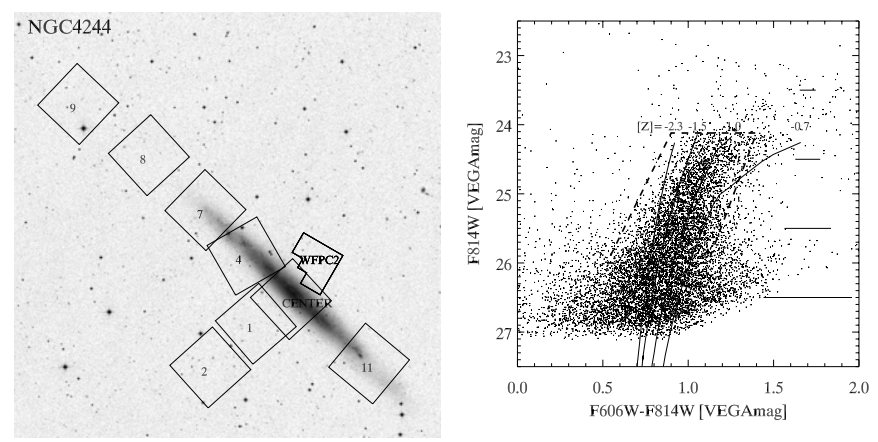

Figure 1. Left - Digitized Sky Survey image of NGC 4244 overlaid with existing HST imaging. Numbered fields show Cycle 14 GHOSTS data, here we analyze the three minor axis ACS fields. Right - Color-magnitude diagram of stars more than 1000 pc below the disk. Overplotted are the box used to study the color of the RGB and 10 Gyr Padova isochrones at four metallicities.
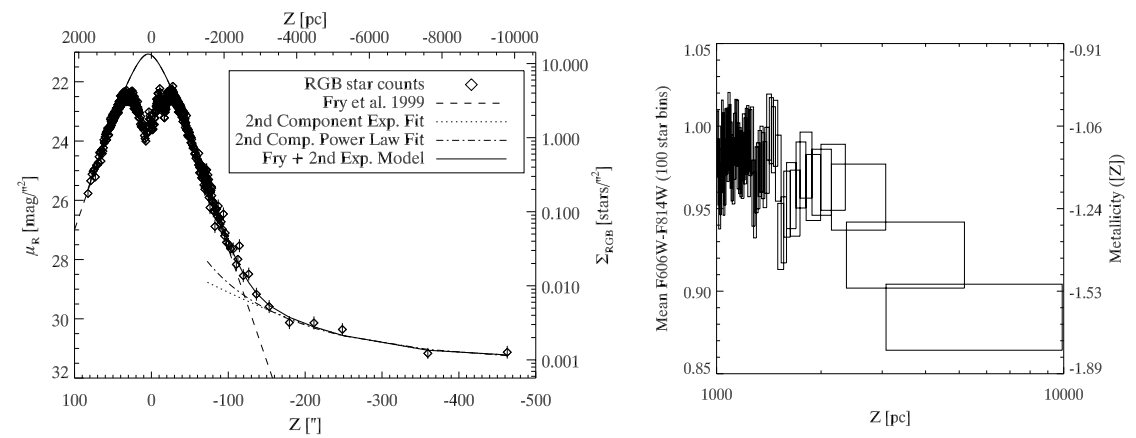

Figure 2. Left - Minor axis profile of NGC 4244. Height above the plane is given in arcseconds (bottom) and parsecs (top). Diamonds show our derived RGB surface density. The dashed line shows the surface photometry from Fry et al. (1999), while other lines show model fits. Right - Mean color vs. disk height for RGB stars. Each box gives the mean color of 100 stars. Box width indicates the disk height of stars, and box height gives the error on the mean color. Equivalent metallicity (right axis) was determined using synthetic CMDs.

Assuming the 2nd component is old, the color of the RGB component is dependent on metallicity (Fig. 1, right panel). The mean color of RGB stars as a function of disk height is shown in the right panel of Fig. 2. To convert mean color to metallicity we used synthetic CMDs. The mean metallicity in the disk component is $[\mathrm{Z}] \sim-1.1$, consistent with the findings of Seth et al. (2005b). However, above $2.5 \mathrm{kpc}$, where the $2 \mathrm{nd}$ component dominates, the metallicity drops to $\sim-1.5$. While this result is intriguing, it should be viewed with caution, as a better knowledge of the contaminants' colors is necessary.

Conclusions: We find strong evidence for a second stellar component along the minor axis of NGC 4244. Both the morphology and metallicity of this second component suggest that it is indeed a stellar halo, similar, but much fainter than that of the Milky Way.

\section{References}

Bullock, J. S., \& Johnston, K. V. 2005, ApJ, 635, 931

Fry, A. M., Morrison, H. L., Harding, P., \& Boroson, T. A. 1999, AJ, 118, 1209

Kalirai, J. S., et al. 2006, ApJ, 648, 389

McConnachie, A. W., et al. 2006, ApJL, 647, L25

Seth, A. C., Dalcanton, J. J., \& de Jong, R. S. 2005a, AJ, 129, 1331

-. 2005b, AJ, 130, 1574

Zibetti, S., White, S. D. M., \& Brinkmann, J. 2004, MNRAS, 347, 556 